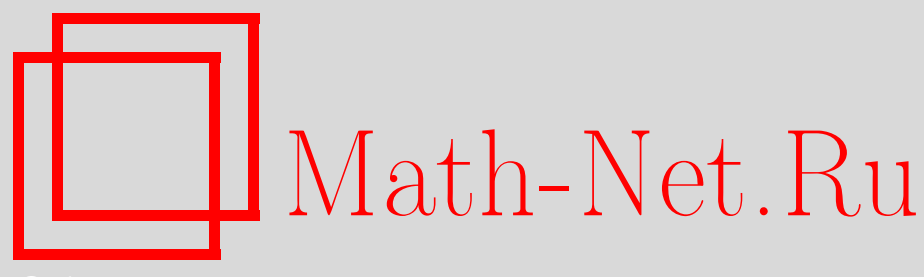

Д. С. Агеев, И. Я. Арефьева, Пробуждение и скремблинг в процессе голографического нагрева, ТМФ, 2017, том 193, номер 1, 146-161

DOI: https://doi.org/10.4213/tmf9337

Использование Общероссийского математического портала Math-Net.Ru подразумевает, что вы прочитали и согласны с пользовательским соглашением http: //www . mathnet.ru/rus/agreement

Параметры загрузки:

IP : 34.229 .108 .108

26 апреля 2023 г., 18:18:31

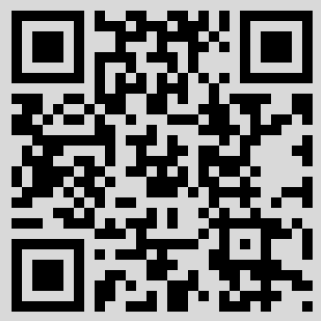




\title{
ФИЗИКА
}

Том 193, № 1

октябрь, 2017

(C) 2017 г.

Д. С. Агеев*, И. Я. Арефьева*

\section{ПРОБУЖДЕНИЕ И СКРЕМБЛИНГ В ПРОЦЕССЕ ГОЛОГРАФИЧЕСКОГО НАГРЕВА}

\begin{abstract}
Методами голографии изучается процесс нагрева в квантовой теории поля. В качестве голографического двойника данного процесса используется поглощение тонкой оболочки безмассовой материи черной браной. Найден явный вид временно́й эволюции квантовой взаимной информации для системы двух интервалов при нагреве от температуры $T_{\mathrm{i}}$ до температуры $T_{\mathrm{f}}$ в двумерном пространстве-времени. Определены геометрические характеристики системы, при которых зависимость взаимной информации от времени имеет вид колокола: в начальный момент времени она равна нулю, в некоторый последующий момент времени становится положительной, далее достигает своего максимума и снова уменьшается до нуля. Такое поведение взаимной информации имеет место в процессе фотосинтеза. Показано, что если расстояние $x$ между интервалами меньше $\ln 2 / 2 \pi T_{\mathrm{i}}$, то эволюция голографической взаимной информации имеет форму колокола лишь при ограниченных сверху и снизу значениях длин интервалов. Для достаточно больших $x$, т. е. при $x>\ln 2 / 2 \pi T_{\mathrm{i}}$, колоколообразная форма зависимости квантовой взаимной информации от времени присутствует только для достаточно больших интервалов, причем эта зона сужается при повышении $T_{\mathrm{i}}$ и расширяется при увеличении $T_{\mathrm{f}}$.
\end{abstract}

Ключевые слова: соответствие АдС/KТП, голография, взаимная информация, энтропия запутанности, скремблинг, пробуждение, нагрев, термализация, фотосинтез.

DOI: https://doi.org/10.4213/tmf9337

\section{1. ВВЕДЕНИЕ}

Соответствие АдС/КТП [1]-[3], изначально возникшее как двойственность между определенными суперсимметричными калибровочными теориями и суперструнами, является мощным инструментом для описания широкого класса квантовых теорий с сильным взаимодействием. В рамках голографического подхода исследовался широкий спектр явлений в КХД [4]-[6], конденсированных средах [7], космологии [8] и даже биологии [9].

Исследование выполнено за счет гранта Российского научного фонда (проект № 14-11-00687).

* Математический институт им. В. А. Стеклова Российской академии наук, Москва, Россия. E-mail: ageev@mi.ras.ru, arefeva@mi.ras.ru 
Один из наиболее трудных вопросов в квантовой теории поля с точки зрения традиционных методов - это описание процесса термализации. В рамках соответствия АдС/КТП температуре в квантовой теории поля сопоставляется температура черной дыры (или черной браны) в двойственном бэкграунде [10]-[12]. Термализация на таком языке рассматривается как процесс образования черной дыры (см. работы [4], [5], а также ссылки приведенные в них). Одно из простейших голографических описаний данного процесса дается деформацией Вадьи некоторой фоновой метрики (см. работы [13]-[21] и ссылки в них).

В настоящей статье мы исследуем процесс внезапного нагрева, используя методы голографии. Такой процесс может служить моделью внезапного возмущения открытой квантовой системы (см., например, [22]), в частности биологических систем (фотосинтез) [9], [23]-[25]. Альтернативное описание процесса нагрева отвечает поглощению черной дырой безмассовой оболочки, а соответствующая метрика описывает черную брану с оболочкой Вадьи. В контексте фотосинтеза особый интерес представляет временна́я эволюция взаимной информации, которая имеет форму колокола. В процессе такой эволюции взаимная информация в начальный момент времени равна нулю, далее становится положительной в момент времени $t_{\text {wup }}$ (мы называем этот момент временем пробуждения), далее возрастает до своего максимума, а затем уменьшается и обращается в нуль в момент времени $t_{\mathrm{scr}}$ (время скремблинга). Данное поведение взаимной информации было найдено численно в голографической модели фотосинтеза [9], при этом использовалась метрика черной дыры с толстой оболочкой Вадьи при частных значениях параметров, определяющих геометрию подсистемы. Наша цель - провести детальный анализ тех значений параметров, при которых эволюция взаимной информации имеет форму колокола, и найти соответствующие этим параметрам время пробуждения $t_{\text {wup }}$ и время скремблинга $t_{\mathrm{scr}}$. Для этого полезно иметь явную формулу зависимости энтропии запутанности от времени вместо численных результатов для толстых оболочек [18], [19], [26], [21], [27]. Мы обобщаем явные результаты, полученные для тонкой оболочки АдС-Вадьи в работах [13], [14], [16], [17], на случай модели черной браны с тонкой оболочкой Вадьи. Явные формулы зависимости от времени для энтропии запутанности в модели черной браны с оболочкой Вадьи являются более громоздкими, чем в случае АдС-Вадьи, однако позволяют существенно упростить детальный анализ эволюции взаимной информации [28], [26], [29], в частности, найти время пробуждения и скремблинга.

Параметры, при которых зависимость взаимной информации от времени имеет форму колокола, образуют подмножество в множестве значений параметров системы, при которых время скремблинга конечно, но время пробуждения отсутствует. Другими словами, зона колокола является частью зоны, где имеется скремблинг. Зона скремблинга в пространстве геометрических параметров нашей модели зависит только от конечной температуры, в то время как зона колокола зависит как от конечной, так и от начальной температуры.

Статья организована следующим образом. В разделе 2 мы описываем геометрию, двойственную процессу нагрева, и приводим явные формулы для энтропии запутанности. Раздел 3 посвящен изучению эволюции взаимной информации во время нагрева; мы оцениваем зоны скремблинга и колокола в пространстве параметров, а также зависимость времен скремблинга и пробуждения от начальной и конечной температур. 


\section{2. ГЕОМЕТРИЯ ЧЕРНОЙ БРАНЫ С ОБОЛОЧКОЙ ВАДЬИ И ГЕОДЕЗИЧЕСКИЕ}

2.1. Геометрия черной браны с оболочкой Вадьи. Мы рассматриваем черную дыру, коллапсирующую из начального состояния, которое определяется положением горизонта $z_{\mathrm{H}}$, в конечное состояние с горизонтом $z_{\mathrm{h}}$, как двойственную фоновую метрику. Метрика, описывающая оболочку Вадьи на фоне черной браны, дается формулами

$$
\begin{aligned}
& v<0: \quad d s^{2}=\frac{1}{z^{2}}\left(-f_{\mathrm{H}}(z) d t^{2}+\frac{d z^{2}}{f_{\mathrm{H}}(z)}+d x^{2}\right), \quad t=v+z_{\mathrm{H}} \operatorname{arth} \frac{z}{z_{\mathrm{H}}}, \\
& v>0: \quad d s^{2}=\frac{1}{z^{2}}\left(-f_{\mathrm{h}}(z) d t^{2}+\frac{d z^{2}}{f_{\mathrm{h}}(z)}+d x^{2}\right), \quad t=v+z_{\mathrm{h}} \operatorname{arth} \frac{z}{z_{\mathrm{h}}},
\end{aligned}
$$

где функции $f_{\mathrm{H}}$ и $f_{\mathrm{h}}$ определены как

$$
f_{\mathrm{H}}(z)=1-\left(\frac{z}{z_{\mathrm{H}}}\right)^{2}, \quad f_{\mathrm{h}}(z)=1-\left(\frac{z}{z_{\mathrm{h}}}\right)^{2}, \quad z_{\mathrm{h}}<z_{\mathrm{H}} .
$$

Начальная и конечная температуры задаются формулами

$$
T_{\mathrm{i}}=\frac{1}{2 \pi z_{\mathrm{H}}}, \quad T_{\mathrm{f}}=\frac{1}{2 \pi z_{\mathrm{h}}} .
$$

В более компактных обозначениях метрика записывается в виде

$$
d s^{2}=\frac{1}{z^{2}}\left(-f(z, v) d t^{2}+\frac{d z^{2}}{f(z, v)}+d x^{2}\right), \quad f(z, v)=\theta(v) f_{\mathrm{h}}(z)+\theta(-v) f_{\mathrm{H}}(z) .
$$

Эта метрика описывает оболочку, расположенную на кривой $v=0$, и обычно предполагается, что выполнено неравенство $z_{\mathrm{H}}>z_{\mathrm{h}}$. Отметим, что случай $z_{\mathrm{H}}<z_{\mathrm{h}}$ соответствует модели охлаждения [15], нарушающей условие энергодоминантности [17].

\section{2. Геодезические в пространстве черной браны с оболочкой Вадьи.} Действие геодезической, которая соединяет две точки $-\ell / 2$ и $\ell / 2$ в момент времени $t$ на границе $z=0$, в фоновой метрике (1) и в параметризации $z=z(x)$ задается формулой

$$
S(\ell)=\int_{-\ell / 2}^{\ell / 2} d x \frac{\sqrt{Q}}{z}, \quad Q=1-2 v^{\prime} z^{\prime}-f(z, v) v^{\prime 2},
$$

где $z( \pm \ell / 2)=0, v( \pm \ell / 2)=t$. Симметрия задачи предполагает, что $z^{\prime}(0)=v^{\prime}(0)=0$. Введем обозначения $z(0)=z_{*}$ и $v(0)=v_{*}$. В областях $v>0$ и $v<0$ динамическая система, задаваемая формулой (5), имеет два интеграла движения $E_{ \pm}=z^{\prime}+f v^{\prime}$ и $J_{ \pm}=z \sqrt{Q}$, где мы обозначаем интегралы выше оболочки $(v>0)$ через $E_{+}, J_{+}$ и под оболочкой $(v<0)$ - через $E_{-}$и $J_{-}$. Интегралы движения $J_{ \pm}$совпадают в силу независимости $Q$ от $x$ и равны значению $z$ в точке максимума $z_{*}$, т. е. точке, где $z^{\prime}=v^{\prime}=0$. Это приводит к соотношениям

$$
z_{*}=z_{-} \sqrt{1-2 v_{-}^{\prime} z_{-}^{\prime}-f_{\mathrm{h}}(z) v_{-}^{\prime 2}}=z_{+} \sqrt{1-2 v_{+}^{\prime} z_{+}^{\prime}-f_{\mathrm{H}}(z) v_{+}^{\prime 2}} .
$$


Над оболочкой мы также имеем равенство $E_{-}=0$, вытекающее из начальных условий $z^{\prime}(0)=v^{\prime}(0)=0$, т. е.

$$
z_{-}^{\prime}+f_{\mathrm{H}}(z) v_{-}^{\prime}=0
$$

при этом в точке пересечения оболочки интеграл $E$ не сохраняется, $E_{+} \neq E_{-}$. Гео-

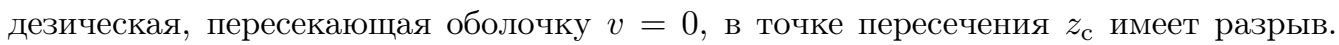
В этой точке мы можем написать равенство для $E_{+}$:

$$
z_{+\mathrm{c}}^{\prime}+f_{\mathrm{H}}\left(z_{\mathrm{c}}\right) v_{+\mathrm{c}}^{\prime}=z_{+}^{\prime}+f_{\mathrm{H}}(z) v_{+}^{\prime},
$$

где $z_{+c}^{\prime}$ обозначает, что мы подходим к точке пересечения из области выше оболочки. Аналогичное обозначение мы используем и для других переменных. Интегралы движения $J_{ \pm}$могут быть сшиты в точке $z_{\mathrm{c}}$,

$$
\begin{aligned}
& \left.z_{-} \sqrt{1-2 v_{-}^{\prime} z_{-}^{\prime}-f_{\mathrm{H}}(z) v_{-}^{\prime 2}}\right|_{z=z_{\mathrm{c}}}=z_{-} \sqrt{1-2 v_{-}^{\prime} z_{-}^{\prime}-f_{\mathrm{H}}(z) v_{-}^{\prime 2}}, \\
& \left.z_{+} \sqrt{1-2 v_{+}^{\prime} z_{+}^{\prime}-f_{\mathrm{h}}(z) v_{+}^{\prime 2}}\right|_{z=z_{\mathrm{c}}}=z_{+} \sqrt{1-2 v_{+}^{\prime} z_{+}^{\prime}-f_{\mathrm{h}}(z) v_{+}^{\prime 2}},
\end{aligned}
$$

и из уравнения (7) в точке пересечения мы имеем

$$
z_{-c}^{\prime}+f_{\mathrm{H}}\left(z_{\mathrm{c}}\right) v_{-c}^{\prime}=0 .
$$

Интегрируя уравнения движения вдоль оболочки, получаем уравнение непрерывности для производной функции $v$ в точке пересечения:

$$
v_{-c}^{\prime}=v_{+\mathrm{c}}^{\prime} .
$$

Решая систему уравнений (6)-(11), мы можем выразить интеграл движения $E_{+}$ и производные $z_{ \pm}^{\prime}, v_{ \pm}^{\prime}$ через $z_{\text {c }}$ и $z_{*}$. Например, $E_{+}$выражается формулой

$$
E_{+}=-\frac{z_{\mathrm{c}}\left(z_{\mathrm{H}}^{2}-z_{\mathrm{h}}^{2}\right)}{2 z_{\mathrm{H}} z_{\mathrm{h}}^{2}} \sqrt{\frac{z_{*}^{2}-z_{\mathrm{c}}^{2}}{z_{\mathrm{H}}^{2}-z_{\mathrm{c}}^{2}}} .
$$

Введем параметры $s, \rho, \kappa, c$ и $\Delta$ равенствами

$$
z_{*}=\frac{z_{\mathrm{c}}}{s}, \quad z_{\mathrm{c}}=\frac{z_{\mathrm{h}}}{\rho}, \quad z_{\mathrm{H}}=\frac{z_{\mathrm{h}}}{\kappa}, \quad c=\sqrt{1-s^{2}}, \quad \Delta=\sqrt{\rho^{2}-\kappa^{2}} ;
$$

cp. с работой [13], где рассматривался случай $z_{\mathrm{H}}=\infty$, т. е. $\kappa=0$. В этих обозначениях $E_{+}$записывается как

$$
E_{+}=-\frac{c\left(1-\kappa^{2}\right)}{2 \Delta \rho s} .
$$

Выражение для времени $t$ на границе $z=0$ имеет вид

$$
t=\int_{0}^{z_{\mathrm{c}}} \frac{d z}{f_{\mathrm{h}}(z)}\left(\frac{E_{+} z}{\sqrt{\left(z_{*}^{2}-z^{2}\right) f_{\mathrm{h}}(z)+E_{+}^{2} z^{2}}}-1\right) .
$$

Вычисляя интеграл в правой части равенства (14) и подставляя в него $E_{+}$из формулы (13), мы получаем

$$
\operatorname{cth} \frac{t}{z_{\mathrm{h}}}=\frac{\left(-\kappa^{2}+2 \rho^{2}+1\right) c+2 \rho \Delta}{2(\Delta+\rho c)} .
$$


Это выражение дает связь времени на концах геодезической с геометрическими характеристиками геодезической - точкой вершины геодезической $z_{*}$ и точкой $z_{\mathrm{c}}$ пересечения с оболочкой.

Выражение для расстояния $\ell$ между точками на границе, из которых выходит геодезическая, получается следующим образом. Расстояние $\ell$ представляется как сумма двух частей, соответствующих двум различным частям геодезической, над и под оболочкой. Мы получаем $\ell=2\left(\ell_{-}+\ell_{+}\right)$, где $\ell_{-}$и $\ell_{+}$задаются в явном виде формулами

$$
\frac{\ell_{-}}{2}=\int_{z_{\mathrm{c}}}^{z_{*}} \frac{z^{2} d z}{\sqrt{z^{2}\left(z_{*}^{2}-z^{2}\right) f_{\mathrm{H}}(z)}}, \quad \frac{\ell_{+}}{2}=\int_{0}^{z_{\mathrm{c}}} \frac{z^{2} d z}{\sqrt{z^{2}\left(z_{*}^{2}-z^{2}\right) f_{\mathrm{h}}(z)+E_{+}^{2} z^{4}}} .
$$

Интегрируя явно в (16) и вводя обозначение $\gamma^{2}=1-\kappa^{2}$, мы получаем искомые формулы:

$$
\begin{aligned}
\frac{\ell_{-}}{2} & =\frac{z_{\mathrm{h}}}{2 \kappa} \ln \frac{\rho^{2} s^{2}-\kappa^{2}}{(c \kappa+\Delta s)^{2}}, \\
\frac{\ell_{+}}{2} & =\frac{z_{\mathrm{h}}}{2} \ln \frac{c^{2} \gamma^{4}-4 \Delta\left(\Delta\left(\rho^{2}-2\right) s^{2}+\Delta-c s \sqrt{4 \Delta^{2}\left(\rho^{2}-1\right)+\gamma^{4}}\right)}{c^{2} \gamma^{4}-4 \Delta^{2}(\rho s-1)^{2}}
\end{aligned}
$$

и

$$
\ell(s, \rho)=2\left(\ell_{-}(s, \rho)+\ell_{+}(s, \rho)\right) .
$$

Длины каждой части геодезической имеют вид

$$
\begin{aligned}
\frac{\mathcal{L}_{-}}{2} & =z_{*} \int_{z_{\mathrm{c}}}^{z_{*}} \frac{d z}{z^{2} \sqrt{\left(1-z^{2} / z_{\mathrm{H}}^{2}\right)\left(z_{*}^{2} / z^{2}-1\right)}}, \\
\frac{\mathcal{L}_{+, \varepsilon}}{2} & =z_{*} \int_{\varepsilon}^{z_{\mathrm{c}}} \frac{d z}{z^{2} \sqrt{\left(1-z^{2} / z_{\mathrm{h}}^{2}\right)\left(z_{*}^{2} / z^{2}-1\right)+E_{+}^{2}}},
\end{aligned}
$$

где мы ввели регуляризацию $\varepsilon$ для расходящейся длины части геодезической $\mathcal{L}_{\varepsilon}$. После интегрирования и стандартного вычитания расходящейся части из $\mathcal{L}_{+, \varepsilon}$ при стремлении $\varepsilon \rightarrow 0$ мы получаем

$$
\frac{\mathcal{L}_{-}}{2}=\frac{1}{2} \ln \frac{\rho\left(c^{2} \rho+2 c \Delta+\rho\right)-\kappa^{2}}{\Delta^{2}-c^{2} \rho^{2}}, \quad \frac{\mathcal{L}_{+}}{2}=\ln \frac{2 \Delta z_{\mathrm{h}}}{\sqrt{\mathcal{K}_{-\mathcal{S}} \mathcal{K}_{+\mathcal{S}}}},
$$

где

$$
\mathcal{K}_{ \pm \mathcal{S}}=2 \Delta(\rho \pm 1)-c\left(\kappa^{2}-2 \rho(\rho \pm 1)-1\right) .
$$

Голографическая энтропия запутанности для одного интервала дается длиной геодезической, натянутой на этот интервал [30]. В явном виде (см. (12)-(20)) мы имеем

$$
S(s, \rho)=2\left(\mathcal{L}_{-}(s, \rho)+\mathcal{L}_{+}(s, \rho)\right) .
$$

Нам, однако, нужна зависимость $S$ от $t$ и $\ell$. Эти переменные в свою очередь явно зависят от $s$ и $\rho$ (см. соотношения (15) и (18)). Наша задача состоит в том, чтобы явно выразить $s$ и $\rho$ через $t$ и $\ell$, т. е. получить функции $s=s(\ell, t)$ и $\rho=\rho(\ell, t)$, и подставить 
их в $(21)$, в результате мы будем иметь зависимость $S(\ell, t)=S(s(t, \rho), \rho(t, \ell))$. Чтобы найти $s=s(t, \ell)$ и $\rho=\rho(t, \ell)$, нам нужно обратить выражения $\ell=\ell(s, \rho)$ и $t=t(s, \rho)$, где $\ell(s, \rho)$ дается формулой (18), а $t=t(s, \rho)$ - формулой (15). Формула (15) позволяет свести нахождение $\rho$ при заданных $s$ и $t$ к решению алгебраического уравнения. Подставляя $\rho=\rho(s, t)$ в $\ell(s, \rho)$, мы получаем функцию $\ell=\ell(s, t)$, которую обращаем численно, находя таким образом $s=s(t, \ell)$.

\section{3. ЭВОЛЮЦИЯ ВЗАИМНОЙ ИНФОРМАЦИИ}

3.1. Виды эволюции взаимной информации. Теперь рассмотрим эволюцию взаимной информации. Взаимная информация для системы двух непересекающихся интервалов длины $\ell_{1}$ и $\ell_{2}$, разделенных расстоянием $x$ в момент времени $t$, задается формулой

$$
I\left(\ell_{1}, \ell_{2}, x, t\right)=S\left(\ell_{1}, t\right)+S\left(\ell_{2}, t\right)-\left(S\left(\ell_{1}+\ell_{2}+x, t\right)+S(x, t)\right) .
$$

Поведение взаимной информации во время термализации было исследовано в работах [28], [26], где рассматривалась модель АдС-Вадьи. Мы изучаем общие характеристики поведения взаимной информации в модели черной дыры с оболочкой Вадьи, т. е. когда начальное состояние уже является термальным. Квантовый квенч в квантовой теории, изначально находящейся в термальном состоянии, изучался в работе [31].

Интерес для нас представляет один из видов эволюции взаимной информации, а именно, когда зависимость взаимной информации от времени имеет вид колокола. Как было отмечено в работе [9], такой тип поведения голографической взаимной информации воспроизводит результаты численных вычислений для квантовых систем, описывающих фотосинтез [32].

Существуют четыре различных режима эволюции взаимной информации во время нагрева системы двух интервалов:

- режим, при котором взаимная информация начинает эволюционировать с ненулевого значения, далее возрастает и достигает своего максимума $I_{\max }$, а затем убывает до фиксированного ненулевого $I_{\min } \neq 0$ (тонкая сплошная линия на рис. 1 показывает этот тип эволюции взаимной информации);

- режим с точкой скремблинга, т. е. режим, при котором в момент времени $t_{\mathrm{scr}}$ взаимная информация становится равной нулю (штриховая линия на рис. 1);

- режим, при котором в момент времени $t_{\text {wup }}$ взаимная информация начинает эволюцию с нулевого значения (пунктирная кривая на рис. 1);

- режим с нулевой взаимной информацией (жирная линия на рис. 1).

Эти режимы имеют такой же вид, как и эволюция взаимной информации в процессе термализации [28], [26], [29], и их физическое объяснение аналогично модели термализации.

На рис. 2 приведена эволюция взаимной информации для различных конечных и начальных температур при разных и одинаковых размерах двух интервалов. Мы видим, что для одинаковых интервалов время пробуждения меньше, а время скремблинга больше.

Далее мы рассматриваем равные отрезки, $\ell_{1}=\ell_{2}=\ell$. В этом случае множество значений параметров двумерно - это область переменных $(x, \ell), \ell>0, x \geqslant 0$. Она 


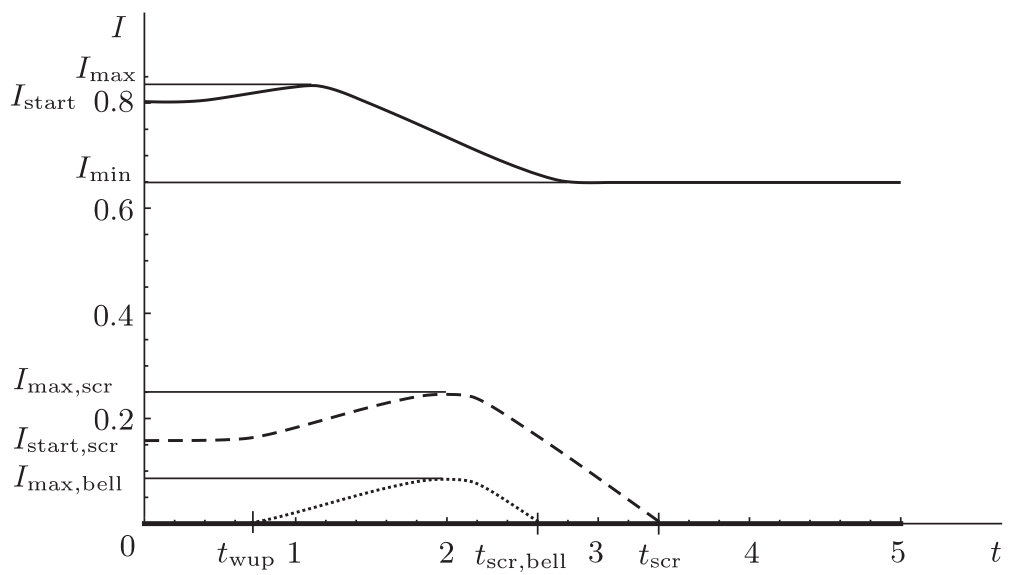

Рис. 1. Различные режимы поведения взаимной информации в процессе нагрева для системы двух непересекающихся интервалов. Тонкая сплошная кривая начинается при ненулевом значении взаимной информации $I_{\text {start }}$, затем возрастает и достигает своего максимума $I_{\max }$, после этого убывает до некоторого фиксированного значения $I_{\min } \neq 0$. Штриховая кривая соответствует режиму со скремблингом, $I\left(t_{\mathrm{start}, \mathrm{scr}}\right) \neq 0, I\left(t_{\mathrm{scr}}\right)=0$. Пунктирная кривая соответствует режиму колокола, т. е. $I\left(t_{\mathrm{wup}}\right)=I\left(t_{\mathrm{scr}, \mathrm{bell}}\right)=0$ и $t_{\mathrm{wup}}<t_{\mathrm{scr}, \text { bell. }}$ Жирная линия соответствует взаимной информации, равной нулю во время всего процесса нагрева.

может быть разделена на четыре зоны, в каждой из которых реализуется один из четырех типичных режимов, изображенных на рис. 3. Очевидно, что при достаточно больших $x$ и фиксированных $\ell$ взаимная информация тождественно равна нулю, как и для больших $\ell$ и достаточно малых $x$ (см. рис. 1$)$. Режим со скремблингом начинается при таких значениях параметров системы, для которых взаимная информация стационарной системы обращается в нуль при конечной температуре. Кривая в плоскости $(x, \ell)$, на которой взаимная информация тождественно равна нулю при температуре $T_{\mathrm{f}}$, задается следующим уравнением:

$$
\ell\left(x, z_{\mathrm{h}}\right)=2 z_{\mathrm{h}} \operatorname{arth}\left(\sqrt{2} \operatorname{sh}\left(\frac{x}{2 z_{\mathrm{h}}}\right)^{-1}-\operatorname{cth}\left(\frac{x}{2 z_{\mathrm{h}}}\right)\right),
$$

где температура $T_{\mathrm{f}}$ выражается через $z_{\mathrm{h}}$ стандартным образом, см. формулу (4).

Для малых $x$ мы имеем разложение

$$
\ell=(1+\sqrt{2}) x+\frac{24+17 \sqrt{2}}{24 z_{\mathrm{h}}^{3}} x^{3}+\mathcal{O}\left(x^{5}\right) .
$$

При больших $\ell$ эта кривая стремится слева к значению $x_{\mathrm{scr}}$,

$$
x \underset{\ell \rightarrow \infty}{\longrightarrow} x_{\mathrm{scr}}=z_{\mathrm{h}} \ln 2 .
$$

В момент $0<t \ll \ell / 2$ температура системы определяется величиной $z_{\mathrm{H}}$, и линия, разделяющая область значений параметров, при которых эволюция взаимной 

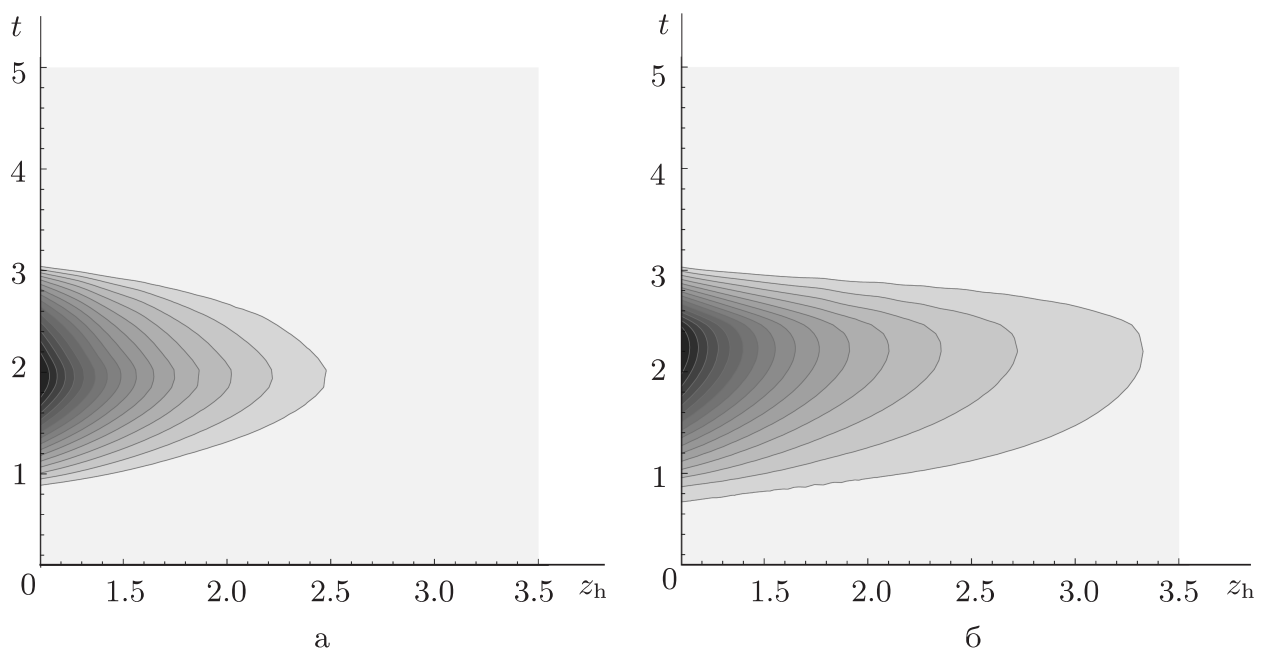

Рис. 2. Графики линий уровня взаимной информации системы двух интервалов для фиксированной начальной температуры $z_{\mathrm{H}}=4$ и переменной конечной температуры: случай отрезков разной длины $\ell_{1}=4, \ell_{2}=6$ (a) и случай отрезков одинаковой длины $\ell_{1}=\ell_{2}=5$ (б) при $x=1.68$. Самая светлая внешняя область соответствует нулевой взаимной информации.

информации имеет вид колокола, и область, где присутствует только режим скремблинга, задается формулой $(22)$ с $z_{\mathrm{H}}$ вместо $z_{\mathrm{h}}$, таким образом, $x_{\mathrm{wup}}=z_{\mathrm{H}} \ln 2$ при больших $\ell$.

На рис. 3 представлено общее расположение зон скремблинга и колокола.

\section{2. Конфигурации, соответствующие колоколообразной эволюции вза-} имной информации. В этом пункте мы подробно изучаем область значений параметров $\ell$ и $x$, при которых в процессе нагрева имеет место колоколообразная эволюция взаимной информации. Как упоминалось ранее, в этом случае для системы двух непересекающихся интервалов (с параметрами $\ell, x$ ) взаимная информация в начальный момент времени равна нулю, затем в момент времени $t_{\text {wup }}$ становится положительной и далее обращается в нуль в момент времени $t_{\mathrm{scr}}$. Мы изучаем зависимость времени скремблинга и времени пробуждения от $\ell$ и $x$.

На рис. 4 изображена зависимость времени пробуждения (штриховая линия) и времени скремблинга (сплошная линия) от расстояния $x$ между интервалами при фиксированных размерах интервалов для различных начальных и конечных температур. На рис. 4а конечная температура зафиксирована, и мы видим, что существует критическое значение $x_{\mathrm{cr}, \operatorname{wup}}\left(z_{\mathrm{H}}\right)$, из которого начинаются при $t=0$ кривые для времени пробуждения. Значения $x_{\mathrm{cr} \text {,wup }}\left(z_{\mathrm{H}}\right)$ не зависят от $z_{\mathrm{h}}$ и выполнены неравенства $x_{\mathrm{cr} \text {,wup }}\left(z_{\mathrm{H}}\right)<x_{\text {wup }}(t)<x_{0}\left(z_{\mathrm{H}}, z_{\mathrm{h}}\right)$. При расстоянии, равном $x_{0}\left(z_{\mathrm{h}}, z_{\mathrm{H}}\right)$, время пробуждения исчезает, а время скремблинга появляется. Линии, соответствующие скремблингу, удовлетворяют неравенствам $x_{\mathrm{cr}, \mathrm{scr}}\left(z_{\mathrm{h}}\right)<x_{\mathrm{scr}}(t)<x_{0}\left(z_{\mathrm{H}}, z_{\mathrm{h}}\right)$. Мы видим, что для больших значений $z_{\mathrm{H}}$ мы получаем бо́льшие значения $t_{\mathrm{scr}}$ и меньшие

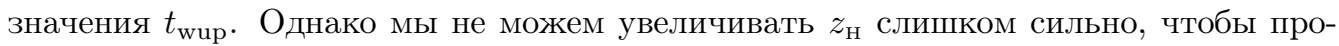
извольно уменьшать $t_{\mathrm{wup}}$, так как для фиксированного $x$ существует критическое значение $x_{\mathrm{cr}, \mathrm{wup}}\left(z_{\mathrm{H}}\right)$. 

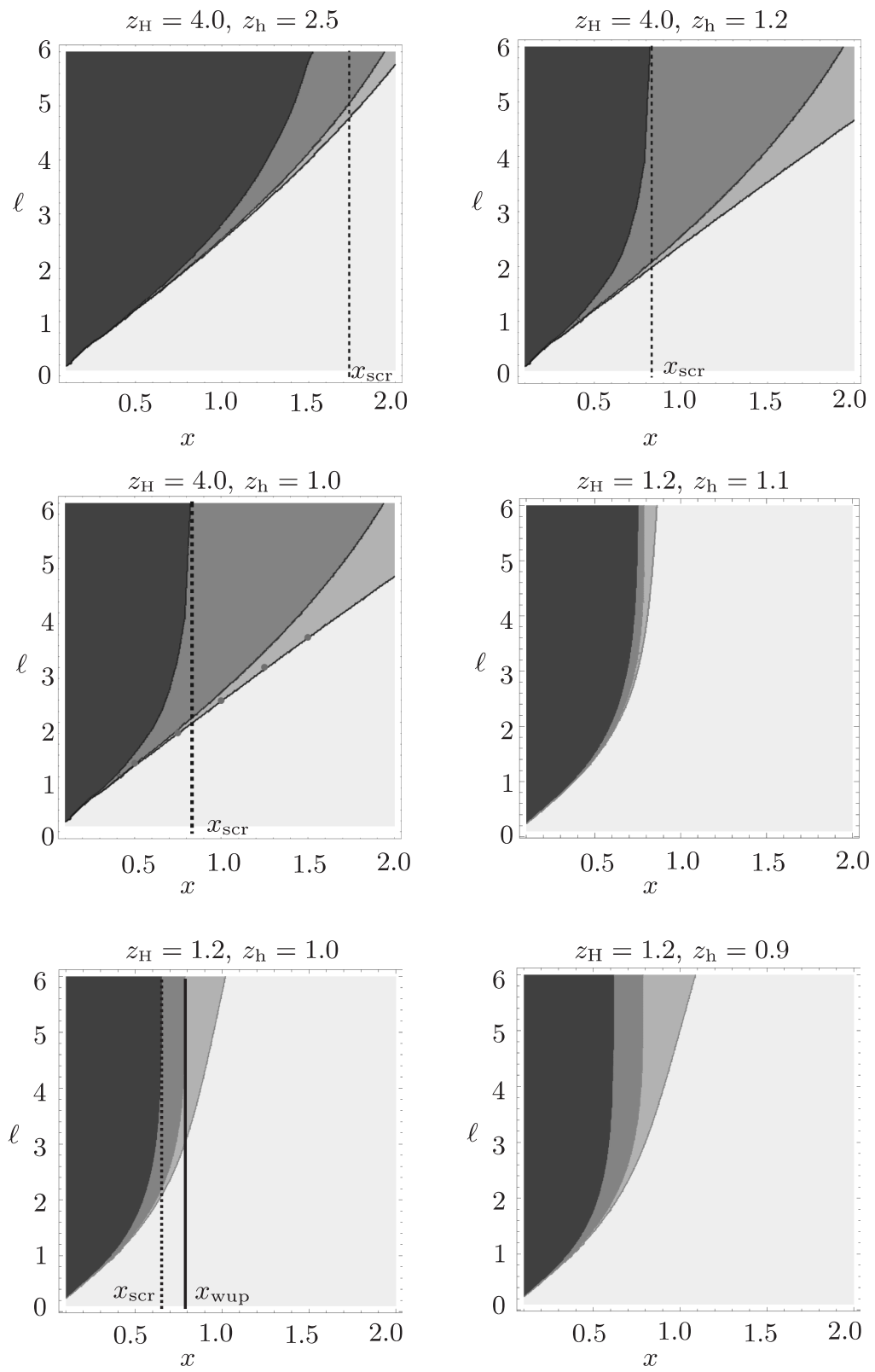

Рис. 3. Зоны различных режимов поведения взаимной информации при различных размерах интервалов $\ell$ и расстояниях $x$ между ними. Вертикальная сплошная прямая соответствует критическому значению $x_{\mathrm{scr}}=z_{\mathrm{h}} \ln 2$, при котором присутствует скремблинг, а вертикальная пунктирная прямая соответствует критическому значению $x_{\text {wup }}=z_{\mathrm{H}} \ln 2$. Различные оттенки цвета соответствуют различным режимам, представленным на рис. 1. 
$z_{\mathrm{h}}=1, \ell=4$

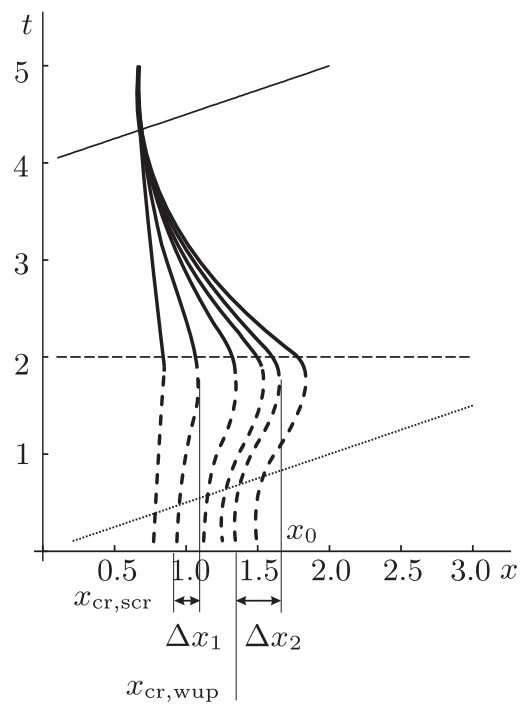

a
$z_{\mathrm{H}}=4, \ell=4$

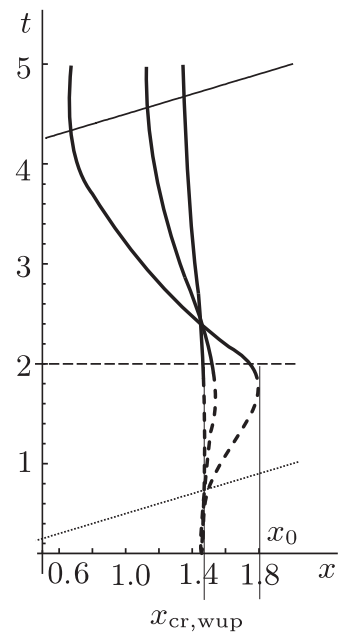

6
$z_{\mathrm{H}}=4, \ell=6$

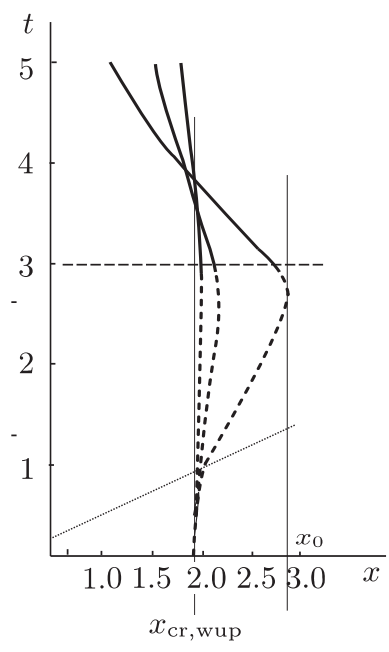

B

Рис. 4. Зависимость времен скремблинга и пробуждения от расстояния $x$ между интервалами. На графике "а" приведена зависимость времени скремблинга (сплошные кривые) и времени пробуждения (штриховые кривые) при различных начальных температурах $\left(z_{\mathrm{H}}=1.2,1.5,2.0,2.5,3.0,5.0\right.$ слева направо) и при фиксированной конечной температуре $z_{\mathrm{h}}=1$. На графиках "б" и "в" приведены время скремблинга (сплошные линии) и время пробуждения (штриховые линии) при различных конечных температурах $\left(z_{\mathrm{h}}=1.0,2.0,3.0\right.$ слева направо, если смотреть на верхнюю часть рисунков) и при фиксированной $z_{\mathrm{H}}=4$. Размеры интервалов равняются $\ell=4$ (а, б) и $\ell=6$ (в). Пунктирная, штриховая и сплошная прямые на каждом графике соответствуют времени термализации отрезков длины $x, \ell$ и $x+\ell$ соответственно.

Видно, что при малой разнице между конечной и начальной температурой в очень узком интервале $\Delta x$ мы можем получить одновременно время скремблинга и пробуждения, т. е. расстояние между двумя вертикальными линиями на рис. 4а убывает, когда убывает разность температур. Например, на рис. 4а выполняется неравенство $\Delta x_{1}<\Delta x_{2}$. Другими словами, больший сигнал, т. е. бо́льшая разница температур, допускает обмен информацией для более широкого диапазона параметров. Также мы видим на рис. $4 \mathrm{a}$, что $x_{\mathrm{cr}, \mathrm{scr}}$ зависит от $z_{\mathrm{h}}$ и не зависит от $z_{\mathrm{H}}$. Из рис. $4 б$ и 4в

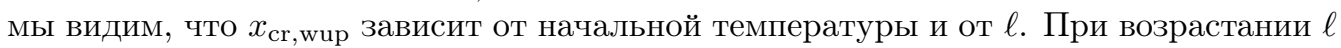
также возрастают величины $x_{\mathrm{cr}, \text { wup }}, x_{0}$ и $x_{0}-x_{\mathrm{cr}, \mathrm{wup}}$.

На рис. 5 изображена зависимость времени скремблинга и времени пробуждения от размера интервалов $\ell_{1}=\ell_{2}=\ell$ при фиксированной начальной температуре $z_{\mathrm{H}}=4$ и различных конечных температурах. Мы видим, что время пробуждения существует для интервалов, удовлетворяющих неравенству $\ell_{0}<\ell<\ell_{\mathrm{cr}, \mathrm{scr}}$, и что 


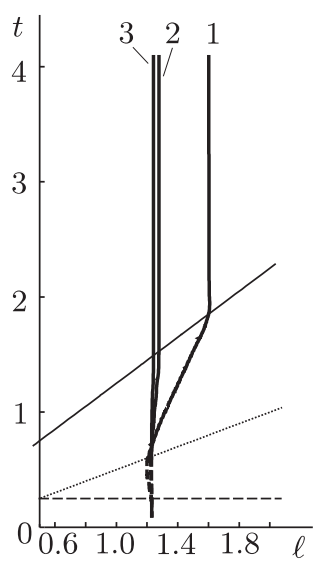

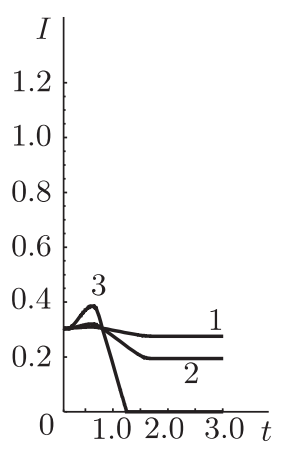

a

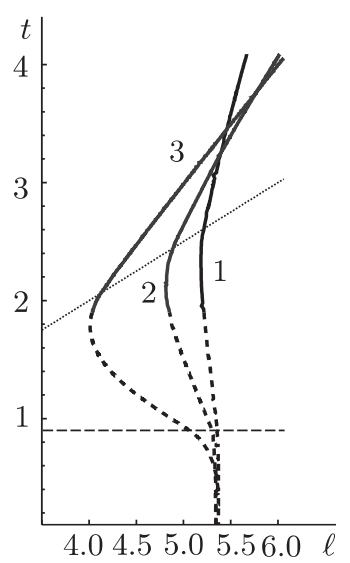

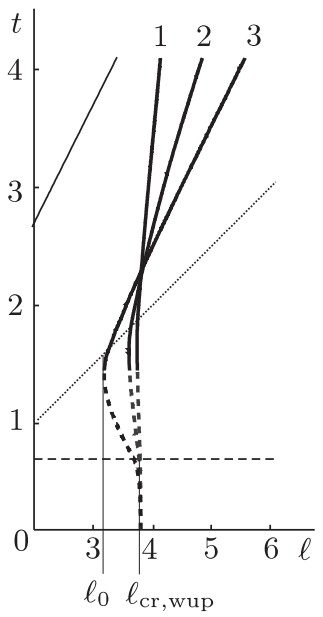

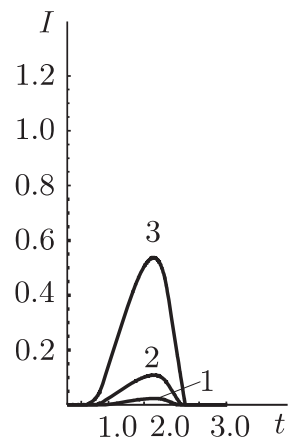

б

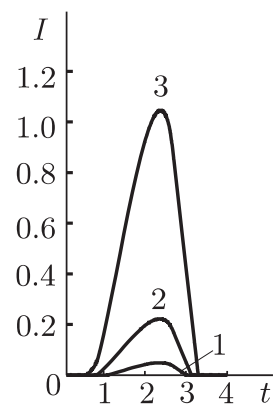

Рис. 5. Зависимость времени скремблинга (сплошные линии) и времени пробуждения (штриховые линии) от $\ell$ при фиксированной начальной температуре $z_{\mathrm{H}}=4$ и различных конечных температурах $z_{\mathrm{h}}=1$ (кривая 1 ), $z_{\mathrm{h}}=2$ (кривая 2), $z_{\mathrm{h}}=3$ (кривая 3) для $x=0.5, \ell=1.4$ (a), $x=1.4, \ell=3.8$ (б) и $x=1.8, \ell=5.3$ (в). Пример эволюции взаимной информации, соответствующей некоторым значениям параметров, представлен справа от каждого графика.

возрастание расстояния $x$ между интервалами приводит к увеличению $\ell_{0}, \ell_{\mathrm{cr}, \mathrm{scr}}$ и $\ell_{\mathrm{cr}, \mathrm{scr}}-\ell_{0}$.

На рис. 6 изображена зависимость времени скремблинга и времени пробуждения от длины интервалов $\ell_{1}=\ell_{2}=\ell$ при $z_{\mathrm{h}}=1$ и различных начальных температурах. Мы видим, что если $z_{\mathrm{H}}>z_{\mathrm{cr}}\left(z_{\mathrm{h}}\right), z_{\mathrm{cr}}(1)=1.47$, то существует критическое значение $\ell_{\mathrm{cr}, \mathrm{wup}}=\ell_{\mathrm{cr}, \mathrm{wup}}\left(z_{\mathrm{H}}\right)$ и значение $\ell_{0}\left(z_{\mathrm{H}}\right)$, такие что только в диапазоне $\ell_{0}<\ell \leqslant \ell_{\mathrm{cr}, \mathrm{wup}}$ существует время пробуждения. Время скремблинга существует при $\ell_{0}<\ell$. Таким 


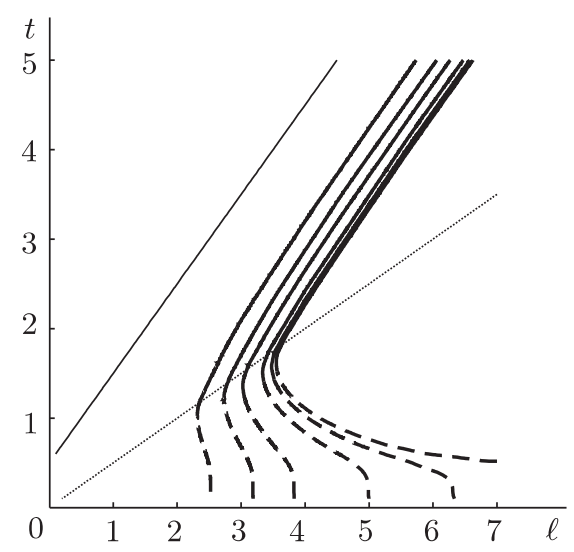

a
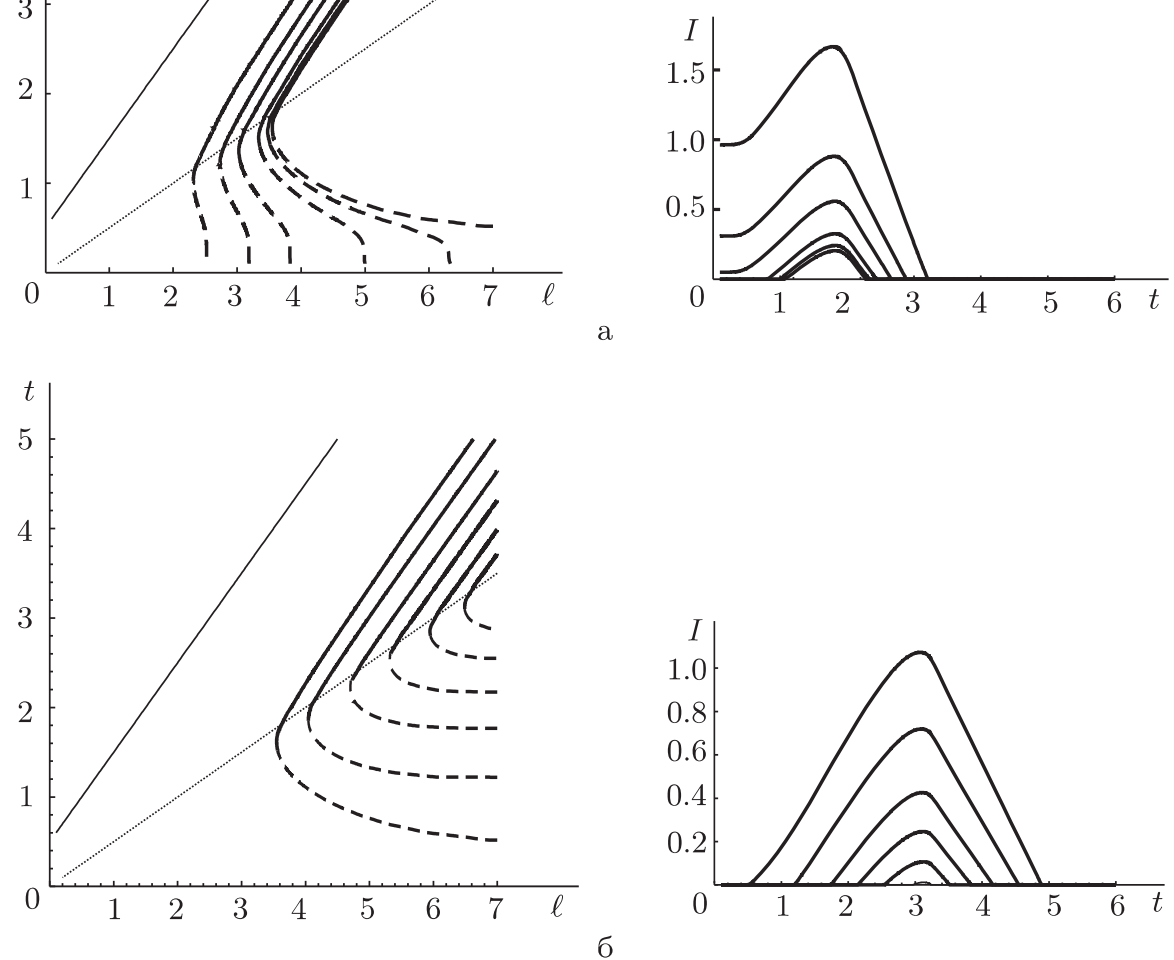

Рис. 6. Зависимость времени скремблинга (сплошные линии) и времени пробуждения (штриховые линии) от $\ell$ при различных начальных температу$\operatorname{pax} z_{\mathrm{H}}$ (большие графики) и эволюция взаимной информации при соответствующих большим графикам значениях парамеров (малые графики). На графике "а" кривые отвечают $z_{\mathrm{H}}=4.5,2.0,1.7,1.54,1.49,1.47$ слева направо для большого графика и сверху вниз для малого графика. На графике "б" кривые отвечают $z_{\mathrm{H}}=1.47,1.37,1.3,1.26,1.23,1.21$ слева направо и сверху вниз. Отметим, что кривая, отвечающая $z_{\mathrm{H}}=1.47$ (пятая из шести на графике "а" и первая слева на графике "б") одна и та же. Сплошная, штриховая и пунктирная прямые линии на каждом из рисунков соответствуют времени термализации для интервала длины $2 l+x$, длины $l$ и длины $x$. Графики построены для $x=1$ и $z_{\mathrm{h}}=1$.

образом, для температур $z_{\mathrm{H}}>z_{\mathrm{cr}}\left(z_{\mathrm{h}}\right)$ область значений параметров, при которых зависимость взаимной информации от времени имеет вид колокола, находится в диа-

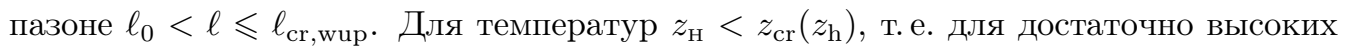
начальных температур (но меньших, чем конечная), колоколообразное поведение имеет место всегда при достаточно больших $\ell$. 


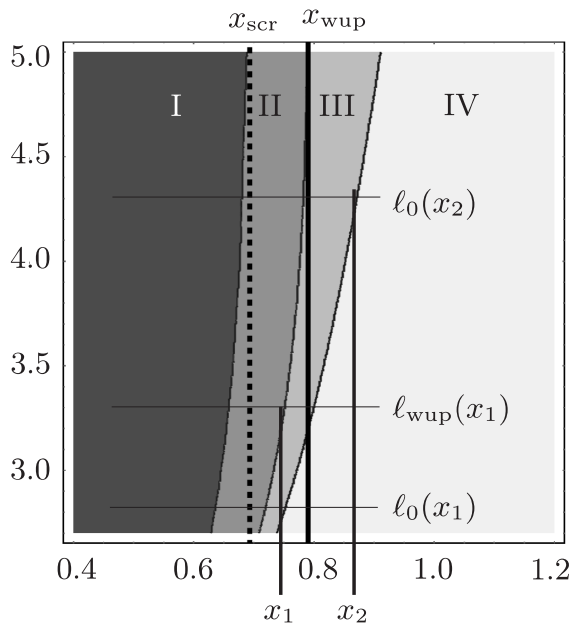

a

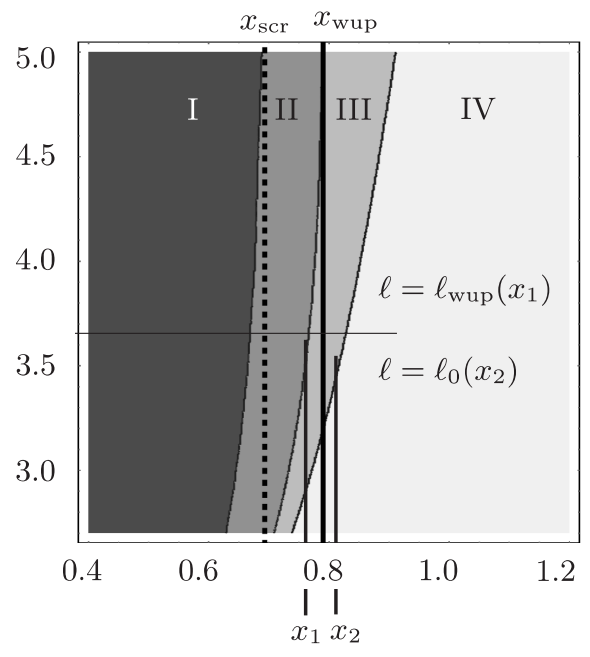

б

Рис. 7. Зоны различных режимов поведения взаимной информации для $z_{\mathrm{H}}=1.2$ и $z_{\mathrm{h}}=1.0$ при различных $\ell$ и $x$ : точки $\left(x_{1}, \ell_{\mathrm{wup}}\left(x_{1}\right)\right.$ находится на границе зон II и III (a); точки $\left(x_{2}, \ell_{0}\left(x_{2}\right)\right)$ находятся на границе зон III и IV (б).

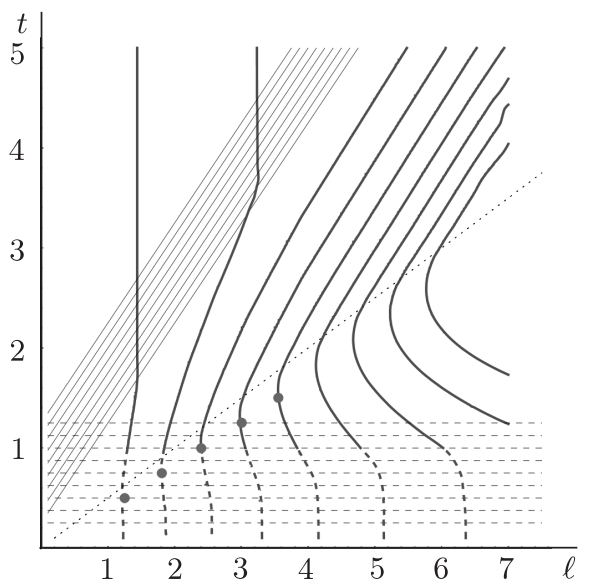

a

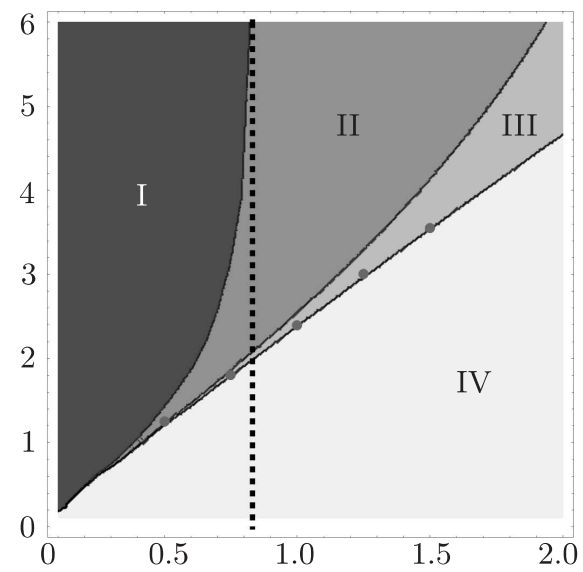

б

Рис. 8. Зависимость времени обращения в ноль взаимной информации от параметра $\ell$. Расстояние между отрезками фиксировано для каждой кривой (а). Точки на границе зон III и IV на графике "б" и точки на графике "а" находятся в однозначном соответствии. Начальные и конечные горизонты фиксированы при $z_{t H}=4.0, z_{\mathrm{h}}=1.2$. 
На рис. 4-6 можно видеть, как реализуется зонная структура, представленная на рис. 3. Рассмотрим рис. 7, показывающий часть рис. 3. На правом графике зафиксируем $x_{1}<z_{\mathrm{H}} \ln 2$. Мы всегда можем найти значение $\ell_{0}=\ell_{0}\left(x_{1}\right)$ такое, что при возрастании $\ell$ при $\ell>\ell_{0}$ мы находимся в зоне колоколообразной эволюции вплоть до $\ell=\ell_{\text {wup }}\left(x_{1}\right)$. Однако если $x=x_{2}>z_{\mathrm{H}} \ln 2$, то зона колоколообразной эволюции начинается в точке $\ell_{0}=\ell_{0}\left(x_{2}\right)$ и существует, пока выполняется условие $\ell>\ell_{0}$. Если мы фиксируем $\ell$, то всегда найдутся $x_{1}$ и $x_{2}$ такие, что $\ell=\ell_{\text {wup }}\left(x_{1}\right)$ и $\ell=\ell_{0}\left(x_{2}\right)$, т. е. точки $\left(x_{1}, \ell_{\text {wup }}\left(x_{1}\right)\right.$ и $\left(x_{2}, \ell_{0}\left(x_{2}\right)\right)$ находятся на границе зон II и III и зон III и IV соответственно, и для всех $x$, удовлетворяющих условию $x_{1}<x<x_{2}$, зависимость взаимной информации от времени имеет вид колокола.

На рис. 8 можно увидеть соответствие между временем, при котором взаимная информация равна нулю, и различными значениями параметров $\ell$ и $x$ (взятых на границе режима, когда взаимная информация тождественно обращается в ноль).

\section{4. ЗАКЛЮЧЕНИЕ}

В представленной статье, используя соответствие АдС/КТП, мы изучили различные аспекты поведения голографической взаимной информации в процессе нагрева. В качестве начального состояния было выбрано термальное состояние, а в качестве дуальной фоновой метрики, описывающей процесс нагрева, была взята метрика черной дыры с оболочкой Вадьи в приближении тонкой оболочки. Получены явные формулы для геодезических в этом $(2+1)$-мерном фоне и далее описана эволюция взаимной информации для системы двух непересекающихся интервалов. В нашей следующей работе [33], используя формулы для голографической энтропии запутанности, мы получили явное описание различных режимов процесса голографического нагрева.

Мы уделили особое внимание случаю, когда зависимость взаимной информации от времени имеет вид колокола. Этот интерес мотивирован важной ролью, которую эволюция такого типа играет в процессе фотосинтеза [32]. Наиболее широкая зона колокола соответствует симметричным конфигурациям и большой разности между начальной и конечной температурой. Эта зона существует при малых расстояниях $x<z_{\mathrm{H}} \ln 2$, а также для определенных значений $\ell$, которые удовлетворяют неравенству $\ell_{\mathrm{scr}}(x)<\ell<\ell_{0}(x)$, и для достаточно больших значений $x$ : когда $x>z_{\mathrm{H}} \ln 2$ и $\ell>\ell_{\mathrm{scr}}(x)=\ell\left(x, z_{\mathrm{H}}\right)$, где $\ell\left(x, z_{\mathrm{H}}\right)$ задается формулой $(22)$.

Понятие квантовой взаимной информации помогает понять квантовые структуры, возникающие в процессе фотосинтеза. Тот факт, что как результат глобального квенча появляется колоколообразная временна́я зависимость взаимной информации в температурных состояниях, дает возможность обмена информацией между двумя подсистемами даже при ненулевой начальной температуре. В контексте фотосинтеза это может быть интерпретировано как отправка сигнала от антенны к реакционному центру. Эволюция типа колокола при непрерывном нагреве была найдена численно в работе [9]. Было бы интересно изучить такую же задачу для $3 \leqslant n \leqslant 7$ отрезков, особенно для трехмерного обобщения на области, имеющие форму шара, а также для случаев более сложных фоновых метрик из работ [16], [34]-[36].

Благодарности. Мы хотели бы поблагодарить И. В. Воловича и М. А. Храмцова за полезные обсуждения. 


\section{Список литературы}

[1] J. M. Maldacena, "The Large $N$ limit of superconformal field theories and supergravity", Adv. Theor. Math. Phys., 2:2 (1998), 231-252, arXiv: hep-th/9711200.

[2] S. S. Gubser, I. R. Klebanov, A. M. Polyakov, "Gauge theory correlators from noncritical string theory", Phys. Lett B., 428:1-2 (1998), 105-114, arXiv: hep-th/9802109.

[3] E. Witten, "Anti-de Sitter space and holography", Adv. Theor. Math. Phys., 2:2 (1998), 253-291, arXiv: hep-th/9802150.

[4] И.Я. Арефьева, "Голографическое описание кварк-глюонной плазмы, образующейся при столновениях тяжелых ионов", УФН, 184:6 (2014), 569-598; I. Aref'eva, "Multiplicity and theremalization time in heavy-ions collisions", Europhys. J. Web Conf., 125 (2016), 01007, 12 pp.

[5] O. DeWolfe, S. S. Gubser, C. Rosen, D. Teaney, "Heavy ions and string theory", Prog. Part. Nucl. Phys., 75 (2014), 86-132, arXiv: 1304.7794.

[6] J. Casalderrey-Solana, H. Liu, D. Mateos, K. Rajagopal, U. A. Wiedemann, Gauge/string duality, hot QCD and heavy ion collisions, arXiv: 1101.0618.

[7] S. A. Hartnoll, "Lectures on holographic methods for condensed matter physics", Class. Quantum Grav., 26:22 (2009), 224002, 61 pp., arXiv: 0903.3246.

[8] R. Easther, R. Flauger, P. McFadden, K. Skenderis, "Constraining holographic inflation with WMAP", JCAP, 09 (2011), 030, 20 pp., arXiv: 1104.2040.

[9] I. Ya. Aref'eva, I. Volovich, Holographic photosynthesis, arXiv: 1603.09107.

[10] E. Witten, "Anti-de Sitter space, thermal phase transition, and confinement in gauge theories", Adv. Theor. Math. Phys., 2:3 (1998), 505-532, arXiv: hep-th/9803131.

[11] J. M. Maldacena, "Eternal black holes in anti-de Sitter", JHEP, 04 (2003), 021, 17 pp., arXiv: hep-th/0106112.

[12] U.H. Danielsson, E. Keski-Vakkuri, M. Kruczenski, "Black hole formation in AdS and thermalization on the boundary", JHEP, 02 (2000), 039, 21 pp., arXiv: hep-th/9912209.

[13] V. Balasubramanian, A. Bernamonti, J. de Boer, N. Copland, B. Craps, E. Keski-Vakkuri, B. Müller, A. Schäfer, M. Shigemori, W. Staessens, "Holographic thermalization", Phys. Rev. D, 84:2 (2011), 026010, 31 pp., arXiv: 1103.2683.

[14] J. Aparício, E. López, "Evolution of two-point functions from holography", JHEP, 12 (2011), 082, 34 pp., arXiv: 1109.3571.

[15] I. Ya. Arefeva, I. V. Volovich, On holographic thermalization and dethermalization of quark-gluon plasma, arXiv: 1211.6041; И.Я. Арефьева, И. В. Волович, "О голографической термализации", ТМФ, 174:2 (2013), 216-227.

[16] I. Aref'eva, A. Bagrov, A.S. Koshelev, "Holographic thermalization from Kerr-AdS", JHEP, 07 (2013), 170, 15 pp., arXiv: 1305.3267.

[17] H. Liu, S. J. Suh, "Entanglement tsunami: Universal scaling in holographic thermalization", Phys. Rev. Lett., 112:1 (2014), 011601, arXiv: 1305.7244.

[18] T. Albash, C. V. Johnson, "Evolution of holographic entanglement entropy after thermal and electromagnetic quenches", New J. Phys., 13 (2011), 045017, 27 pp., arXiv: 1008.3027.

[19] V. Keränen, E. Keski-Vakkuri, L. Thorlacius, "Thermalization and entanglement following a non-relativistic holographic quench", Phys. Rev. D, 85:2 (2012), 026005, 14 pp., arXiv: 1110.5035 .

[20] И. Я. Арефьева, "Время образования кварк-глюонной плазмы при столкновениях тяжелых ионов в голографической модели с ударными волнами", ТМФ, 184:3 (2015), 398-417.

[21] I. Ya. Aref'eva, A. A. Golubtsova, E. Gourgoulhon, "Analytic black branes in Lifshitz-like backgrounds and thermalization", JHEP, 09 (2016), 142, 36 pp., arXiv: 1601.06046.

[22] M. Ohya, I. Volovich, Mathematical Foundations of Quantum Information and Computation and Its Applications to Nano- and Bio-Systems, Springer, Dordrecht, 2011. 
[23] И. Я. Арефьева, И. В. Волович, С. В. Козырев, "Метод стохастического предела и интерференция в квантовых многочастичных системах", ТМФ, 183:3 (2015), 388-408.

[24] И. В. Волович, С. В. Козырев, “Манипуляция состояниями вырожденной квантовой системы", Тр. МИАН, 294 (2016), 256-267.

[25] I. V. Volovich, "Cauchy-Schwarz inequality-based criteria for the non-classicality of sub-Poisson and antibunched light", Phys. Lett. A, 380:1-2 (2016), 56-58.

[26] A. Allais, E. Tonni, "Holographic evolution of the mutual information", JHEP, 01 (2012), 102, arXiv: 1110.1607.

[27] D. Ageev, "Holographic Wilson loops in anisotropic quark-gluon plasma", Europhys. J. Web Conf., 125 (2016), 04007, 6 pp.

[28] V. Balasubramanian, A. Bernamonti, N. Copland, B. Craps, F. Galli, "Thermalization of mutual and tripartite information in strongly coupled two dimensional conformal field theories", Phys. Rev. D, 84:10 (2011), 105017, 7 pp., arXiv: 1110.0488.

[29] M. Alishahiha, M.R. Mohammadi Mozaffar, M. R. Tanhayi, "On the time evolution of holographic n-partite information", JHEP, 09 (2015), 165, 61 pp., arXiv: 1406.7677.

[30] S. Ryu, T. Takayanagi, "Holographic derivation of entaglement entropy from the anti-de Sitter space/conformal field theory", Phys. Rev. Lett., 96:18 (2006), 181602, 4 pp., arXiv: hep-th/0603001.

[31] S. Sotiriadis, P. Calabrese, J. Cardy, "Quantum quench from a thermal initial state", Europhys. Lett., 87:2 (2009), 20002, 6 pp., arXiv: 0903.0895.

[32] K. Bradler, M. M. Wilde, S. Vinjanampathy, D. B. Uskov, "Identifying the quantum correlations in light-harvesting complexes", Phys. Rev. A, 82:6 (2010), 062310, 12 pp., arXiv: 0912.5112 .

[33] D. S. Ageev, I. Ya. Aref'eva, Memory loss in holographic non-equilibrium heating, arXiv: 1704.07747.

[34] D. Galante, M. Schvellinger, "Thermalization with a chemical potential from AdS spaces", JHEP, 07 (2012), 096, 27 pp., arXiv: 1205.1548.

[35] E. Caceres, A. Kundu, "Holographic thermalization with chemical potential", JHEP, 09 (2012), 055, 44 pp., arXiv: 1205.2354.

[36] M. Mezei, "On entanglement spreading from holography", JHEP, 05 (2017), 064, 46 pp., arXiv: 1612.00082 . 